\title{
PARTICLES AND FIELDS
}

\section{RAPID COMMUNICATIONS}

Rapid Communications are intended for important new results which deserve accelerated publication, and are therefore given priority in the editorial office and in production. A Rapid Communication in Physical Review D should be no longer than five printed pages and must be accompanied by an abstract. Page proofs are sent to authors, but because of the accelerated schedule, publication is generally not delayed for receipt of corrections unless requested by the author.

\section{Search for neutrinoless $\tau$ decays: $\tau \rightarrow e \gamma$ and $\tau \rightarrow \mu \gamma$}

K. W. Edwards, ${ }^{1}$ A. Bellerive, ${ }^{2}$ R. Janicek, ${ }^{2}$ D. B. MacFarlane, ${ }^{2}$ K. W. McLean, ${ }^{2}$ P. M. Patel, ${ }^{2}$ A. J. Sadoff, ${ }^{3}$ R. Ammar, ${ }^{4}$ P. Baringer, ${ }^{4}$ A. Bean, ${ }^{4}$ D. Besson, ${ }^{4}$ D. Coppage, ${ }^{4}$ C. Darling, ${ }^{4}$ R. Davis, ${ }^{4}$ N. Hancock, ${ }^{4}$ S. Kotov, ${ }^{4}$ I. Kravchenko, ${ }^{4}$ N. Kwak, ${ }^{4}$ S. Anderson, ${ }^{5}$ Y. Kubota, ${ }^{5}$ M. Lattery, ${ }^{5}$ J. J. O'Neill, ${ }^{5}$ S. Patton, ${ }^{5}$ R. Poling, ${ }^{5}$ T. Riehle, ${ }^{5}$ V. Savinov, ${ }^{5}$ A. Smith, ${ }^{5}$ M. S. Alam, ${ }^{6}$ S. B. Athar, ${ }^{6}$ Z. Ling, ${ }^{6}$ A. H. Mahmood, ${ }^{6}$ H. Severini, ${ }^{6}$ S. Timm, ${ }^{6}$

F. Wappler, ${ }^{6}$ A. Anastassov, ${ }^{7}$ S. Blinov, ${ }^{7, *}$ J. E. Duboscq, ${ }^{7}$ D. Fujino, ${ }^{7, \dagger}$ R. Fulton, ${ }^{7}$ K. K. Gan, ${ }^{7}$ T. Hart, $^{7}$ K. Honscheid, ${ }^{7}$ H. Kagan, ${ }^{7}$ R. Kass, ${ }^{7}$ J. Lee, ${ }^{7}$ M. B. Spencer, ${ }^{7}$ M. Sung, ${ }^{7}$ A. Undrus, ${ }^{7, *}$ R. Wanke, ${ }^{7}$ A. Wolf, ${ }^{7}$ M. M. Zoeller, ${ }^{7}$ B. Nemati, ${ }^{8}$ S. J. Richichi, ${ }^{8}$ W. R. Ross, ${ }^{8}$ P. Skubic,${ }^{8}$ M. Wood, ${ }^{8}$ M. Bishai, ${ }^{9}$ J. Fast, ${ }^{9}$ E. Gerndt, ${ }^{9}$ J. W. Hinson, ${ }^{9}$ N. Menon, ${ }^{9}$ D. H. Miller, ${ }^{9}$ E. I. Shibata, ${ }^{9}$ I. P. J. Shipsey, ${ }^{9}$ M. Yurko, ${ }^{9}$ L. Gibbons, ${ }^{10}$ S. D. Johnson, ${ }^{10}$ Y. Kwon, ${ }^{10}$ S. Roberts, ${ }^{10}$ E. H. Thorndike, ${ }^{10}$ C. P. Jessop, ${ }^{11}$ K. Lingel, ${ }^{11}$ H. Marsiske, ${ }^{11}$ M. L. Perl, ${ }^{11}$ S. F. Schaffner, ${ }^{11}$ D. Ugolini, ${ }^{11}$ R. Wang, ${ }^{11}$ X. Zhou, ${ }^{11}$ T. E. Coan, ${ }^{12}$ V. Fadeyev, ${ }^{12}$ I. Korolkov, ${ }^{12}$ Y. Maravin, ${ }^{12}$ I. Narsky, ${ }^{12}$ V. Shelkov, ${ }^{12}$ J. Staeck, ${ }^{12}$ R. Stroynowski, ${ }^{12}$ I. Volobouev, ${ }^{12}$ J. Ye, ${ }^{12}$ M. Artuso, ${ }^{13}$ A. Efimov, ${ }^{13}$ F. Frasconi, ${ }^{13}$ M. Gao, ${ }^{13}$

M. Goldberg, ${ }^{13}$ D. He, ${ }^{13}$ S. Kopp, ${ }^{13}$ G. C. Moneti, ${ }^{13}$ R. Mountain, ${ }^{13}$ Y. Mukhin, ${ }^{13}$ S. Schuh, ${ }^{13}$ T. Skwarnicki, ${ }^{13}$ S. Stone, ${ }^{13}$

G. Viehhauser, ${ }^{13}$ X. Xing, ${ }^{13}$ J. Bartelt, ${ }^{14}$ S. E. Csorna, ${ }^{14}$ V. Jain, ${ }^{14}$ S. Marka, ${ }^{14}$ A. Freyberger, ${ }^{15}$ D. Gibaut, ${ }^{15}$

R. Godang, ${ }^{15}$ K. Kinoshita, ${ }^{15}$ I. C. Lai, ${ }^{15}$ P. Pomianowski, ${ }^{15}$ S. Schrenk, ${ }^{15}$ G. Bonvicini, ${ }^{16}$ D. Cinabro, ${ }^{16}$ R. Greene, ${ }^{16}$ L. P. Perera, ${ }^{16}$ B. Barish, ${ }^{17}$ M. Chadha, ${ }^{17}$ S. Chan, ${ }^{17}$ G. Eigen, ${ }^{17}$ J. S. Miller, ${ }^{17}$ C. O' Grady, ${ }^{17}$ M. Schmidtler, ${ }^{17}$ J. Urheim, ${ }^{17}$ A. J. Weinstein, ${ }^{17}$ F. Würthwein, ${ }^{17}$ D. M. Asner, ${ }^{18}$ D. W. Bliss, ${ }^{18}$ W. S. Brower, ${ }^{18}$ G. Masek, ${ }^{18}$ H. P. Paar, ${ }^{18}$

J. Gronberg, ${ }^{19}$ R. Kutschke, ${ }^{19}$ D. J. Lange, ${ }^{19}$ S. Menary, ${ }^{19}$ R. J. Morrison, ${ }^{19}$ S. Nakanishi, ${ }^{19}$ H. N. Nelson, ${ }^{19}$ T. K. Nelson, ${ }^{19}$ C. Qiao, ${ }^{19}$ J. D. Richman, ${ }^{19}$ D. Roberts, ${ }^{19}$ A. Ryd, ${ }^{19}$ H. Tajima, ${ }^{19}$ M. S. Witherell, ${ }^{19}$ R. Balest, ${ }^{20}$ B. H. Behrens, ${ }^{20}$ K. Cho, ${ }^{20}$ W. T. Ford, ${ }^{20}$ H. Park, ${ }^{20}$ P. Rankin, ${ }^{20}$ J. Roy, ${ }^{20}$ J. G. Smith, ${ }^{20}$ J. P. Alexander, ${ }^{21}$ C. Bebek, ${ }^{21}$ B. E. Berger, ${ }^{21}$

K. Berkelman, ${ }^{21}$ K. Bloom, ${ }^{21}$ D. G. Cassel, ${ }^{21}$ H. A. Cho, ${ }^{21}$ D. M. Coffman, ${ }^{21}$ D. S. Crowcroft, ${ }^{21}$ M. Dickson, ${ }^{21}$

P. S. Drell, ${ }^{21}$ K. M. Ecklund, ${ }^{21}$ R. Ehrlich, ${ }^{21}$ R. Elia, ${ }^{21}$ A. D. Foland, ${ }^{21}$ P. Gaidarev, ${ }^{21}$ R. S. Galik, ${ }^{21}$ B. Gittelman, ${ }^{21}$ S. W. Gray, ${ }^{21}$ D. L. Hartill, ${ }^{21}$ B. K. Heltsley, ${ }^{21}$ P. I. Hopman, ${ }^{21}$ S. L. Jones, ${ }^{21}$ J. Kandaswamy, ${ }^{21}$ N. Katayama, ${ }^{21}$ P. C. Kim, ${ }^{21}$ D. L. Kreinick, ${ }^{21}$ T. Lee, ${ }^{21}$ Y. Liu, ${ }^{21}$ G. S. Ludwig, ${ }^{21}$ J. Masui, ${ }^{21}$ J. Mevissen, ${ }^{21}$ N. B. Mistry, ${ }^{21}$

C. R. Ng, ${ }^{21}$ E. Nordberg, ${ }^{21}$ M. Ogg, ${ }^{21,+}$ J. R. Patterson, ${ }^{21}$ D. Peterson, ${ }^{21}$ D. Riley, ${ }^{21}$ A. Soffer, ${ }^{21}$ C. Ward, ${ }^{21}$ M. Athanas, ${ }^{22}$ P. Avery, ${ }^{22}$ C. D. Jones, ${ }^{22} \mathrm{M}$. Lohner, ${ }^{22} \mathrm{C}$. Prescott, ${ }^{22} \mathrm{~S}$. Yang, ${ }^{22} \mathrm{~J}$. Yelton, ${ }^{22} \mathrm{~J}$. Zheng, ${ }^{22} \mathrm{G}$. Brandenburg, ${ }^{23}$

R. A. Briere, ${ }^{23}$ Y. S. Gao, ${ }^{23}$ D. Y.-J. Kim, ${ }^{23}$ R. Wilson, ${ }^{23}$ H. Yamamoto, ${ }^{23}$ T. E. Browder, ${ }^{24} \mathrm{~F} . \mathrm{Li}^{24}{ }^{24}$ Y. Li, ${ }^{24}$ J. L. Rodriguez, ${ }^{24}$ T. Bergfeld, ${ }^{25}$ B. I. Eisenstein, ${ }^{25}$ J. Ernst, ${ }^{25}$ G. E. Gladding, ${ }^{25}$ G. D. Gollin, ${ }^{25}$ R. M. Hans, ${ }^{25}$ E. Johnson, ${ }^{25}$ I. Karliner, ${ }^{25}$ M. A. Marsh, ${ }^{25}$ M. Palmer, ${ }^{25}$ M. Selen, ${ }^{25}$ and J. J. Thaler ${ }^{25}$

\author{
(CLEO Collaboration) \\ ${ }^{1}$ Carleton University, Ottawa, Ontario, Canada K1S 5B6 \\ and the Institute of Particle Physics, Canada \\ ${ }^{2}$ McGill University, Montréal, Québec, Canada H3A $2 T 8$ \\ and the Institute of Particle Physics, Canada \\ ${ }^{3}$ Ithaca College, Ithaca, New York 14850 \\ ${ }^{4}$ University of Kansas, Lawrence, Kansas 66045 \\ ${ }^{5}$ University of Minnesota, Minneapolis, Minnesota 55455 \\ ${ }^{6}$ State University of New York at Albany, Albany, New York 12222 \\ ${ }^{7}$ Ohio State University, Columbus, Ohio 43210
}




\author{
${ }^{8}$ University of Oklahoma, Norman, Oklahoma 73019 \\ ${ }^{9}$ Purdue University, West Lafayette, Indiana 47907 \\ ${ }^{10}$ University of Rochester, Rochester, New York 14627 \\ ${ }^{11}$ Stanford Linear Accelerator Center, Stanford University, Stanford, California 94309 \\ ${ }^{12}$ Southern Methodist University, Dallas, Texas 75275 \\ ${ }^{13}$ Syracuse University, Syracuse, New York 13244 \\ ${ }^{14}$ Vanderbilt University, Nashville, Tennessee 37235 \\ ${ }^{15}$ Virginia Polytechnic Institute and State University, Blacksburg, Virginia 24061 \\ ${ }^{16}$ Wayne State University, Detroit, Michigan 48202 \\ ${ }^{17}$ California Institute of Technology, Pasadena, California 91125 \\ ${ }^{18}$ University of California, San Diego, La Jolla, California 92093 \\ ${ }^{19}$ University of California, Santa Barbara, California 93106 \\ ${ }^{20}$ University of Colorado, Boulder, Colorado 80309-0390 \\ ${ }^{21}$ Cornell University, Ithaca, New York 14853 \\ ${ }^{22}$ University of Florida, Gainesville, Florida 32611 \\ ${ }^{23}$ Harvard University, Cambridge, Massachusetts 02138 \\ ${ }^{24}$ University of Hawaii at Manoa, Honolulu, Hawaii 96822 \\ ${ }^{25}$ University of Illinois, Champaign-Urbana, Illinois 61801
}

(Received 12 November 1996)

\begin{abstract}
A search for the lepton-family-number-violating decays $\tau \rightarrow e \gamma$ and $\tau \rightarrow \mu \gamma$ has been performed using CLEO II data. No evidence of a signal has been found and the corresponding upper limits are $B(\tau \rightarrow e \gamma)<2.7 \times 10^{-6}$ and $B(\tau \rightarrow \mu \gamma)<3.0 \times 10^{-6}$ at $90 \%$ C.L. [S0556-2821(97)50207-4]
\end{abstract}

PACS number(s): 13.35.Dx, 11.30.Fs, 14.60.Fg

Nonconservation of the leptonic quantum number is expected in many extensions of the standard model and searches for lepton-number-violating decays provide strong constraints on possible new physics processes. The most stringent limits so far have been obtained in the studies of $\mu$ decays [1]: $B(\mu \rightarrow e \gamma)<4.9 \times 10^{-11}$ and $B(\mu \rightarrow e e e)$ $<1.0 \times 10^{-12}$. Even though we cannot reach a similar level of sensitivity for $\tau$ 's, the search for lepton-number-violating $\tau$ decays becomes competitive with the $\mu$ results in theoretical models with mass-dependent couplings. There have been several recent theoretical calculations based on specific supersymmetric, grand unified theory (GUT) and superstring models [2-4]. For example, a superstring model [4] gives an enhancement of $\tau$ decays over the corresponding $\mu$ decays of $B(\tau \rightarrow \mu \gamma)=2 \times 10^{5} B(\mu \rightarrow e \gamma)$.

Lepton-number-violating neutrinoless $\tau$ decays have been studied extensively. Upper limits have been set by CLEO [5] on branching fractions for 22 channels with three charged particles in the final state at the level of few times $10^{-6}$, and the limit [6] $B(\tau \rightarrow \mu \gamma)<4.2 \times 10^{-6}$ has also been published. This paper describes CLEO's first search for the neutrinoless decay $\tau \rightarrow e \gamma$. The upper limit of $B(\tau \rightarrow e \gamma)$ $<1.2 \times 10^{-4}$ at $90 \%$ C.L. was previously obtained by ARGUS [7]. A new analysis searching for the $\mu \gamma$ final state is also presented.

In this analysis we use data from the reaction $e^{+} e^{-} \rightarrow \tau^{+} \tau^{-}$collected at the Cornell Electron Storage Ring (CESR) at or near the energy of $Y(4 S)$. The data correspond to a total integrated luminosity of $4.68 \mathrm{fb}^{-1}$ and contain

\footnotetext{
*Permanent address: BINP, RU-630090 Novosibirsk, Russia.

†Permanent address: Lawrence Livermore National Laboratory, Livermore, CA 94551.

${ }^{\ddagger}$ Permanent address: University of Texas, Austin, TX 78712.
}

about $4.24 \times 10^{6} \tau^{+} \tau^{-}$pairs. We search for events with a 1-vs-1 topology, where the signal candidate $\tau$ decays into $e \gamma$ or $\mu \gamma$ and the tag side includes all standard $\tau$ decays into one charged particle, any number of photons and at least one neutrino. The selection criteria are based on the studies of two Monte Carlo samples of $10000 \tau$ pair events each. The Monte Carlo sample uses the KORALB [8] generator with two-body phase space for the $e \gamma$ and $\mu \gamma$ decay modes and a detector simulation based on the GEANT package [9].

We select $\tau^{+} \tau^{-}$pair events with exactly two good charged tracks, with total charge equal to zero, and with the angle between the charged tracks greater than $90^{\circ}$. Since radiative Bhabha scattering and $\mu$-pair production provide high background rates, we allow only one identified electron or one identified muon per event. Thus in the $e \gamma$ search, one of the tracks has to be positively identified as an electron while the other should be inconsistent with the electron hypothesis, and in the $\mu \gamma$ search one of the tracks has to be identified as a muon while the other has to be inconsistent with the muon hypothesis. After these criteria are applied, $19.3 \%$ of $e \gamma$ and $36.2 \%$ of $\mu \gamma$ Monte Carlo events remain in the signal region.

In addition, each candidate event must have exactly one photon separated by more than $20^{\circ}$ from the closest charged track in the lepton hemisphere. This photon must lie in a good section of the calorimeter barrel (i.e., $|\cos \theta|<0.71$, where $\theta$ is an angle between the photon and beam directions) and have energy deposition in the calorimeter greater than $300 \mathrm{MeV}$. This minimum energy cut is dictated by the kinematics of two-body $\tau$ decay. The angle between the direction of the photon and the momentum of the electron or the muon track must satisfy $0.4<\cos \theta_{l \gamma}<0.8$, where the upper limit is again dictated by kinematics, and the lower limit by selection efficiency. The Monte Carlo expectation of the $\cos \theta_{e \gamma}$ distribution for the $\tau \rightarrow e \gamma$ channel is compared in Fig. 1 with the 


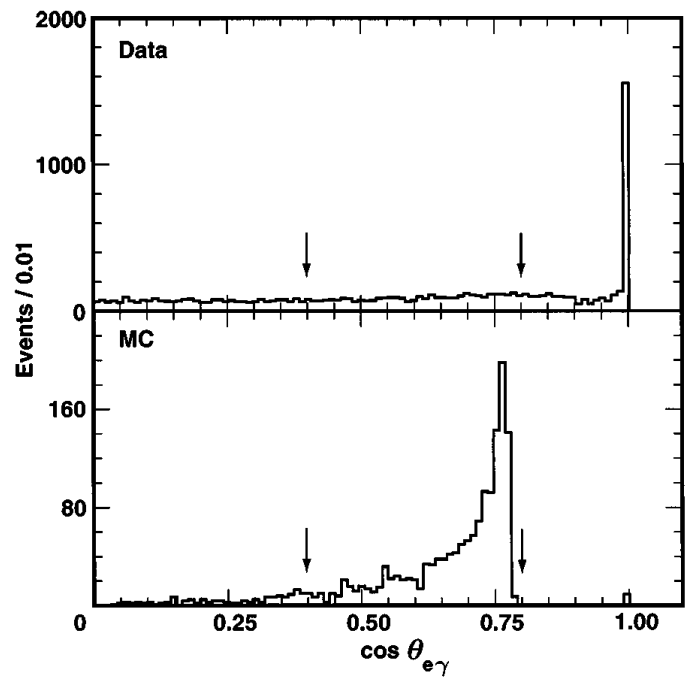

FIG. 1. The $\cos \theta_{e \gamma}$ distribution for $\tau \rightarrow e \gamma$ analysis in data and signal Monte Carlo.

data. The corresponding distributions for $\tau \rightarrow \mu \gamma$ are similar. $14.6 \%$ of the $e \gamma$ and $20.9 \%$ of the $\mu \gamma$ original Monte Carlo sample survive these initial selection criteria.

For electron identification we use both drift chamber $d E / d x$ and calorimeter information. In the $e \gamma$ analysis we require that an electron candidate's specific ionization be within three standard deviations of the expected value, and the energy, $E$, deposited in the calorimeter match the track momentum, $p$, measured in the drift chamber: 0.8 $<E /|p|<1.1$. After these cuts are applied, a large fraction of low momentum electrons, mostly from two photon processes, still survive on the tag side. Therefore, unless the tagging track is identified as a muon, we impose additional requirements to reject soft electrons while keeping hadrons on the tag side: the tagging track's transverse momentum must be greater than $300 \mathrm{MeV} / c$, its momentum must point to the good portion of the calorimeter barrel $(|\cos \theta|<0.71)$, and the $E /|p|$ ratio must be less than 0.6.

In the $\mu \gamma$ analysis a particle is identified as a muon if it traverses at least three absorption lengths of the material, has correlated drift and muon chamber hits, and has a calorimeter response consistent with that of a minimum ionizing particle.

The main sources of background in the selected samples are due to Bhabha scattering, $\mu$-pair production, radiative $\tau \longrightarrow e \gamma \nu \nu$ and $\tau \longrightarrow \mu \gamma \nu \nu$ decays, and two photon processes.

A large fraction of these backgrounds can be rejected by imposing a cut on the angle between the momentum of the tagging particle and the missing momentum of the event. We calculate the missing momentum as a negative of the sum of momenta of the two charged tracks and all showers detected in the calorimeter with energies above $30 \mathrm{MeV}$. Since there must be at least one undetected neutrino on the tag side, the missing momentum in a $\tau$ event is expected to fall into the tagging track hemisphere, while for all radiative processes the missing momentum should be uncorrelated with the charged track on the tag side (see Fig. 2). To reduce this background, we require that the cosine of the angle between the total missing momentum of the event and the momentum of the tagging particle be greater than 0.4 .

The neutrino emission on the tag side should also result in
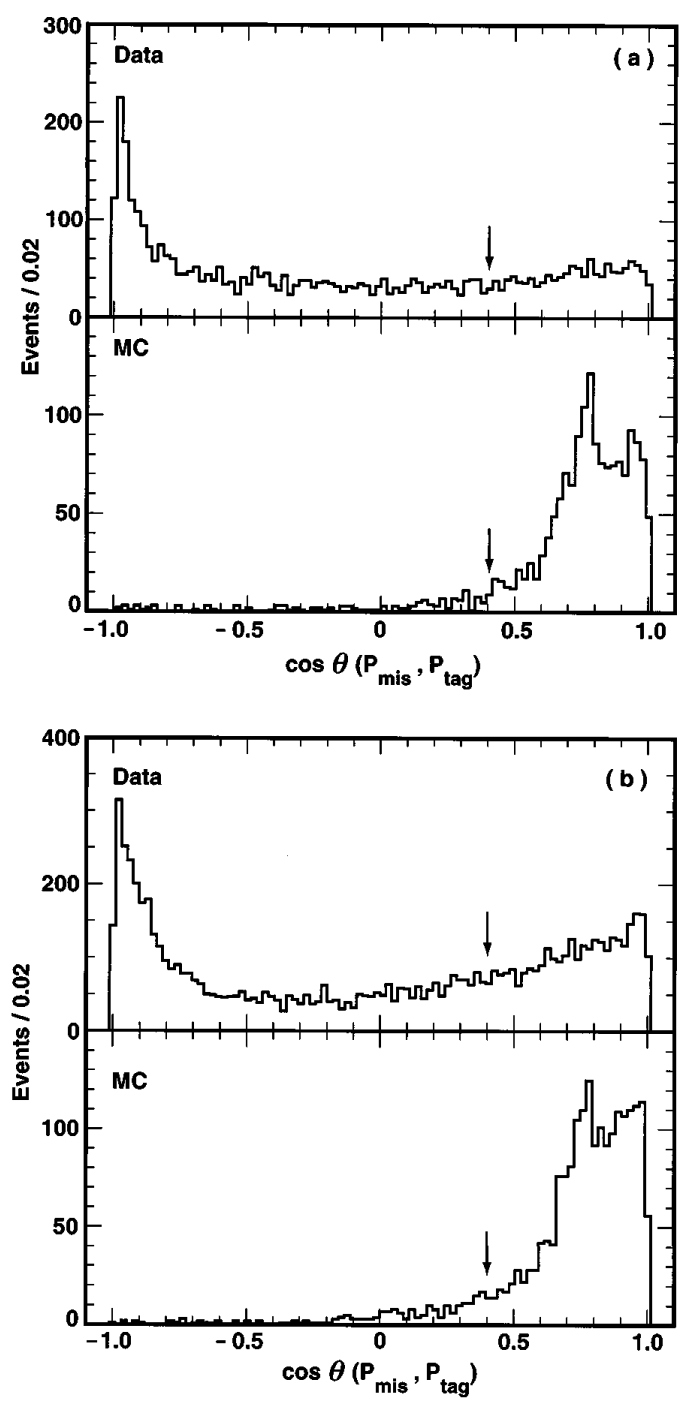

FIG. 2. The cosine of the angle between the missing momentum and the momentum of the tagging track in data and signal Monte Carlo events for (a) $\tau \rightarrow e \gamma$ and (b) $\tau \rightarrow \mu \gamma$. Region $\cos \theta\left(\vec{p}_{\text {mis }}, \vec{p}_{\text {tag }}\right)<0.4$ is rejected.

a large total transverse momentum with respect to the beam direction. The data, however, show a pronounced peak near zero transverse momentum that comes mostly from copious two-photon and radiative QED processes. This background is eliminated by requiring the total transverse momentum of the event to be greater than $300 \mathrm{MeV} / \mathrm{c}$ (see Fig. 3). After all the previous requirements are applied, $13.2 \%$ of $e \gamma$ and $17.9 \%$ of $\mu \gamma$ Monte Carlo sample remain in the signal region.

The final signal selection criteria are based on kinematic constraints since a neutrinoless $\tau$ decay should have a total energy and an effective mass of the $e \gamma$ or $\mu \gamma$ consistent with the beam energy and $\tau$ mass, respectively. To define a signal region in the mass vs energy plane we studied the corresponding Monte Carlo distributions. We fitted the mass and energy distributions separately to a Gaussian function plus a polynomial. The order of the polynomial was increased until a fit with a confidence level above $20 \%$ was obtained. The signal region was then defined to be within \pm 3 standard deviations of the fitted Gaussian component of the distribu- 

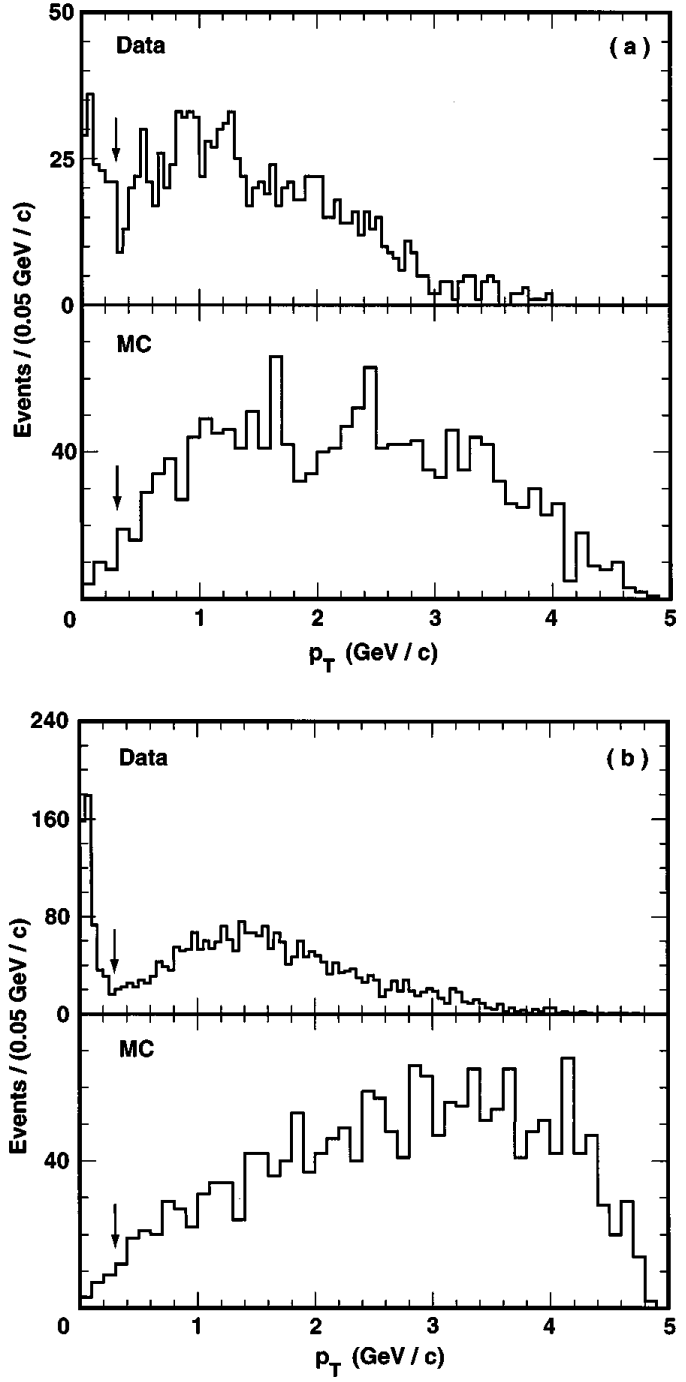

FIG. 3. Total transverse momentum of the event in data and signal Monte Carlo for (a) $\tau \rightarrow e \gamma$ and (b) $\tau \rightarrow \mu \gamma$. Region $p_{T}<0.3 \mathrm{GeV} / c$ is rejected.

tion. In Table I we show mean values of the $\tau$ mass and beam energy and their corresponding resolutions obtained with this fitting technique. The $\pm 3 \sigma$ energy cut was imposed on the difference $\Delta E=E_{l \gamma}-E_{\text {run }}$ between the total energy of lepton and photon and the beam energy of a particular run. The input $\tau$ mass and beam energy in the Monte Carlo samples were $1.777 \mathrm{GeV} / c^{2}$ and $5.29 \mathrm{GeV}$, respectively. After these cuts were applied, no $e \gamma$ and three $\mu \gamma$ events remained in the signal region.

TABLE I. Mean values of effective mass, energy and corresponding resolutions obtained from the fits to Monte Carlo event sample.

\begin{tabular}{lcc}
\hline \hline Channel & $\tau \rightarrow e \gamma$ & $\tau \rightarrow \mu \gamma$ \\
\hline$m_{\tau}\left(\mathrm{GeV} / c^{2}\right)$ & 1.772 & 1.774 \\
$\sigma_{m}\left(\mathrm{GeV} / c^{2}\right)$ & 0.024 & 0.025 \\
$E_{l \gamma}-E_{\text {run }}(\mathrm{GeV})$ & -0.013 & -0.010 \\
$\sigma_{E}(\mathrm{GeV})$ & 0.060 & 0.053 \\
MC efficiency $(\%)$ & 10.1 & 14.4 \\
\hline \hline
\end{tabular}
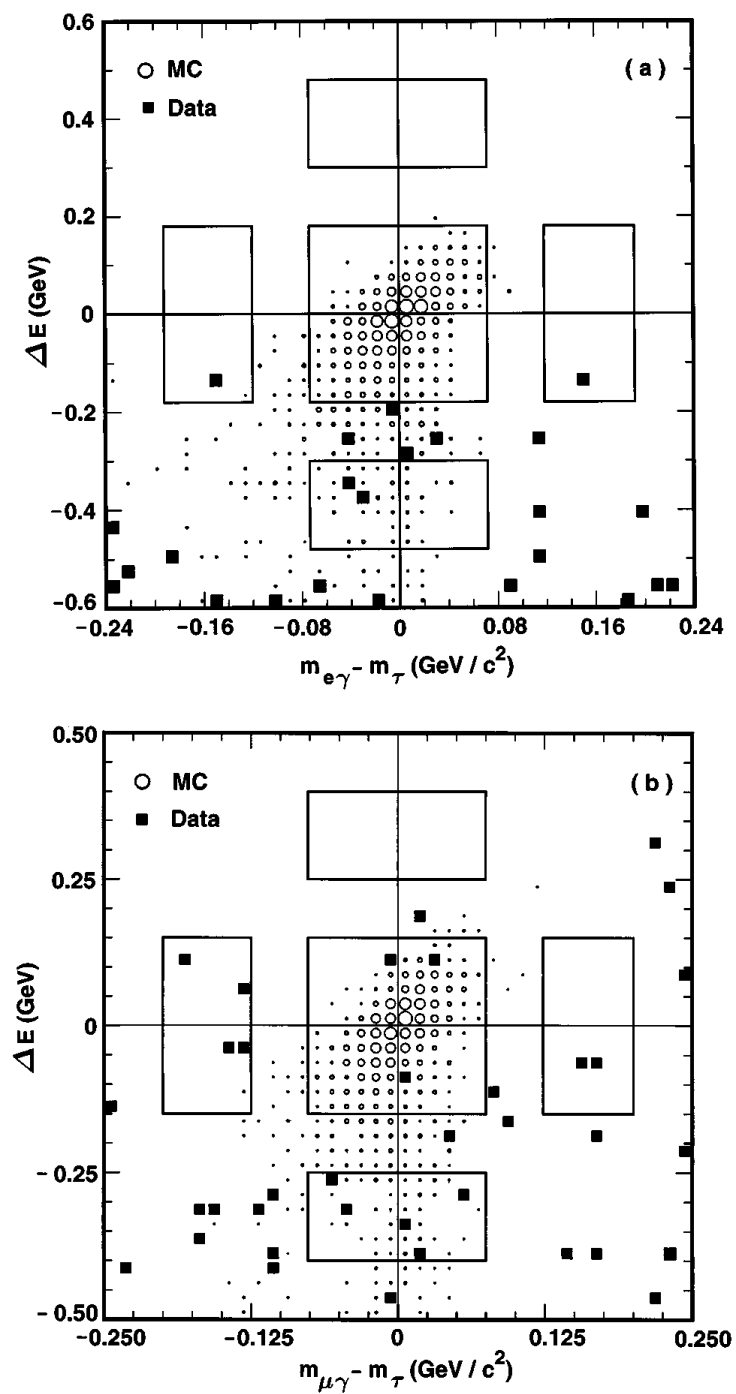

FIG. 4. $\Delta E$ vs $\left(m_{l \gamma}-m_{\tau}\right)$ distributions for (a) $\tau \rightarrow e \gamma$ and (b) $\tau \rightarrow \mu \gamma$. Solid squares represent the data; open circles represent the signal Monte Carlo distributions. The diameter of the circles is proportional to the number of entries. The box at the center represents the signal region, and the four other boxes represent the sideband regions defined in the text.

We estimate the amount of the expected background in each signal region directly from the data by extrapolating it from a sideband region. We assume that the background distributions are linear in the vicinity of $m_{\tau}$ and $\Delta E=0$ and define the sideband regions between five and eight standard deviations as shown in Fig. 4. The region $\left|m_{l \gamma}-m_{\tau}\right|$ $>5 \sigma_{m},|\Delta E|>5 \sigma_{E}$, where $m_{l \gamma}$ is an effective mass of lepton and photon, captures only $4.3 \%$ of the $e \gamma$ and $1.8 \%$ of

TABLE II. Summary of the results.

\begin{tabular}{lcc}
\hline \hline Channel & $\tau \rightarrow e \gamma$ & $\tau \rightarrow \mu \gamma$ \\
\hline MC efficiency, \% & 10.1 & 14.4 \\
$n_{0}$ & 0 & 3 \\
$\mu_{B}$ & 2.0 & 5.5 \\
$\lambda$ & 2.3 & 3.6 \\
Upper limit at 90\% C.L. & $2.7 \times 10^{-6}$ & $3.0 \times 10^{-6}$ \\
\hline \hline
\end{tabular}


the $\mu \gamma$ Monte Carlo samples, so we can neglect the small bias introduced by this extrapolation back into the signal region. The extrapolation from the sidebands allows us to estimate the expected background as 2.0 events for the $e \gamma$ sample and 5.5 events for the $\mu \gamma$ analysis. To check that the background value is stable with respect to the sideband region geometry, we varied the sideband definition. The background estimates were the same within \pm 0.5 events for the $e \gamma$ and \pm 1.0 event for the $\mu \gamma$ channel. Finally, we estimate the background values as $2.0 \pm 0.5$ in the $e \gamma$ and $5.5 \pm 1.0$ in the $\mu \gamma$ analysis. The background rate is higher for the $\mu \gamma$ analysis because the selection criteria for the tagging track are loose, and a large fraction of soft muons that failed the standard identification procedure, mostly from $\mu \mu \gamma$ processes, survive on the tag side. These estimates strongly depend on the assumption of the linearity of the background across the signal region.

To understand the origin of the events remaining in the signal region, we applied our selection criteria to about $27 \times 10^{6}$ continuum hadronic Monte Carlo events and $17 \times 10^{6}$ generic $\tau$ Monte Carlo events. No hadronic Monte Carlo events satisfied the selection requirements. There are 2 e $\gamma$ and $4 \mu \gamma$ events from generic $\tau$ Monte Carlo that survived all the cuts. After normalization to the same luminosity as the data, these correspond to 0.5 and 1.0 event, respectively, and are in reasonable agreement with the numbers of events found in the signal regions. Since the Monte Carlo simulation is uncertain at the level of precision of our measurement, we choose to use background extrapolated from the data for the estimate of upper limits.

Before discussing effects caused by systematic uncertainties, we estimate the upper limits on the branching fractions for the $\tau \rightarrow e \gamma$ and $\tau \rightarrow \mu \gamma$ channels using the statistics of a Poisson process with background [10]:

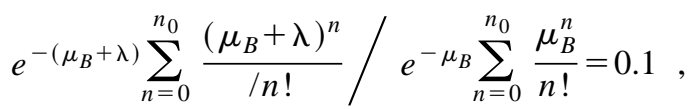

where $\lambda$ is the number of events for the upper limit at $90 \%$ confidence level, $\mu_{B}$ is the expected background, and $n_{0}$ is the number of observed events. The upper limit $L_{U}$ for a branching fraction is:

$$
L_{U}=\lambda / 2 \epsilon N_{\tau \tau},
$$

where $\epsilon$ is the event selection efficiency and $N_{\tau \tau}$ is the total number of $\tau$ pairs produced.
For the $e \gamma$ analysis, $n_{0}=0$ gives us $\lambda=2.3$ events, and the corresponding upper limit is $B(\tau \rightarrow e \gamma)<2.7 \times 10^{-6}$ at $90 \%$ C.L. For the $\mu \gamma$ analysis, $n_{0}=3$ and $\mu_{B}=5.5$ give us the value of $\lambda=3.6$ events, and the corresponding upper limit of $B(\tau \rightarrow \mu \gamma)<2.9 \times 10^{-6}$ at $90 \%$ C.L.

The systematic uncertainty in detector sensitivity $S=2 \epsilon N_{\tau \tau}$ is estimated as $9 \%$ for both $e \gamma$ and $\mu \gamma$ channels. This uncertainty is obtained by adding in quadrature uncertainties in track reconstruction efficiency $(3 \%)$, photon reconstruction efficiency (5\%), cut selection (5\%), luminosity (1.4\%), lepton identification (1.5\% for $e$ and $4 \%$ for $\mu$ ), and Monte Carlo statistics (3\% for $e \gamma$ and $2.5 \%$ for $\mu \gamma$ ). The upper limit for the $\mu \gamma$ channel is also affected by uncertainty in background estimate. To incorporate systematic uncertainties into the upper limits, we assume that the errors related to $\epsilon N_{\tau \tau}$ and to the background estimates have Gaussian distributions and apply a technique described in Ref. [11]. This technique reweights the Poisson probability (1) of observing $\lambda=R \times S$ or a larger number of events by a Gaussian probability density of the detector sensitivity $S$ and a Gaussian probability density of the number of background events $\mu_{B}$. It gives a new value of the upper limit at $90 \%$ C.L.:

$$
\begin{aligned}
\int_{0}^{\infty} \int_{0}^{\infty} e^{-R S} \sum_{n=0}^{n_{0}} \frac{\left(\mu_{B}+R S\right)^{n}}{n !} /\left(\sum_{n=0}^{n_{0}} \frac{\mu_{B}^{n}}{n !}\right) \frac{1}{\sqrt{2 \pi} \sigma_{S}} \\
\quad \times \exp \left[-\left(S-S_{0}\right)^{2} / 2 \sigma_{S}^{2}\right] \frac{1}{\sqrt{2 \pi} \sigma_{B}} \\
\quad \times \exp \left[-\left(\mu_{B}-\mu_{B 0}\right)^{2} / 2 \sigma_{B}^{2}\right] d S d \mu_{B}=0.1,
\end{aligned}
$$

where $R$ is a new upper limit, $S_{0}$ is an unbiased estimate of $S, \mu_{B 0}$ is an estimated value of background, and $\sigma_{S} / S_{0}$ and $\sigma_{B} / \mu_{B 0}$ are relative uncertainties in detector sensitivity and background estimate, respectively. The efficiencies, numbers of events, expected background values, and the recalculated upper limits for the decay branching fractions incorporating systematic errors are summarized in Table II. These results are limited by the total integrated luminosity and represent a significant improvement over previous analyses.

We gratefully acknowledge the effort of the CESR staff in providing us with excellent luminosity and running conditions. This work was supported by the National Science Foundation, the U.S. Department of Energy, the Heisenberg Foundation, the Alexander von Humboldt Stiftung, Research Corporation, the Natural Sciences and Engineering Research Council of Canada, and the A.P. Sloan Foundation.
[1] Particle Data Group, R.M. Barnett et al., Phys. Rev. D 54, 250 (1996).

[2] R. Barbieri and L.J. Hall, Phys. Lett. B 338, 212 (1994).

[3] J. Hisano et al., Phys. Lett. B 357, 579 (1995).

[4] R. Arnowitt and P. Nath, Phys. Rev. Lett. 66, 2708 (1991).

[5] J. Bartelt et al., Phys. Rev. Lett. 73, 1890 (1994).

[6] A. Bean et al., Phys. Rev. Lett. 70, 138 (1993).
[7] H. Albrecht et al., Z. Phys. C 55, 179 (1992).

[8] S. Jadach and Z. Was, Comput. Phys. Commun. 64, 267 (1991); S. Jadach et al., ibid. 76, 361 (1993).

[9] R. Brun et al., GEANT version 3.15, CERN Report No. dd/ee/ 84-1 (unpublished).

[10] Barnett et al. [1], p. 159.

[11] R. Cousins and V. Highland, Nucl. Instrum. Methods Phys. Res. A 320, 331 (1992). 